\title{
A new set of 299 pictures for psycholinguistic studies: French norms for name agreement, image agreement, conceptual familiarity, visual complexity, image variability, age of acquisition, and naming latencies
}

\author{
PATRICK BONIN \\ Université Blaise Pascal, Clermont-Ferrand, France
}

RONALD PEEREMAN

Université de Bourgogne, Dijon, France

and

\author{
NATHALIE MALARDIER, ALAIN MÉOT, and MARYLÈNE CHALARD \\ Université Blaise Pascal, Clermont-Ferrand, France
}

\begin{abstract}
Pictures are often used as stimuli in studies of perception, language, and memory. Since performances on different sets of pictures are generally contrasted, stimulus selection requires the use of standardized material to match pictures across different variables. Unfortunately, the number of standardized pictures available for empirical research is rather limited. The aim of the present study is to provide French normative data for a new set of 299 black-and-white drawings. Alario and Ferrand (1999) were closely followed in that the pictures were standardized on six variables: name agreement, image agreement, conceptual familiarity, visual complexity, image variability, and age of acquisition. Objective frequency measures are also provided for the most common names associated with the pictures. Comparative analyses between our results and the norms obtained in other, similar studies are reported. Finally, naming latencies corresponding to the set of pictures were also collected from French native speakers, and correlational/multiple-regression analyses were performed on naming latencies. This new set of standardized pictures is available on the Internet (http://leadserv.u-bourgogne.fr/bases/pictures/) and should be of great use to researchers when they select pictorial stimuli.
\end{abstract}

Researchers involved in the study of perception, language, and memory processes often use pictures as experimental stimuli (Alario \& Ferrand, 1999; Baddeley, 1990; Gisselbrecht, 1988; Paivio, 1969; Snodgrass \& Vanderwart, 1980). Pictures are idiosyncratic in nature and, thus, vary along several dimensions, such as, for example, their visual complexity (VC), the way they are drawn, and the familiarity of the objects they represent. Also, the labels that can be used to refer to the objects depicted by the pictures have different characteristics. For instance, they vary in their frequency of occurrence in the language and in their age of ac-

The authors thank Laetitia De Rycke for collecting the norms, the students who participated in the different rating tasks, and Roberto Dell' Acqua, Judith Kroll, and Jonathan Vaughan for very helpful comments on a previous version of the paper. Correspondence concerning this article should be addressed to P. Bonin, Laboratoire de Psychologie Sociale de la Cognition et de Psychologie Cognitive(LAPSCO), Université Blaise Pascal, 34, avenue Carnot, 63037 Clermont-Ferrand, France (email: patrick.bonin@srvpsy.univ-bpclermontfr). quisition (AoA). Finally, the relation between the picture and its name is not necessarily unambiguous. A particular picture can evoke different names, and a particular name can evoke different pictorial representations. In order to ensure control over these variations, it is therefore important for researchers who use pictures as stimuli to have normative data at their disposal.

Before the pioneering study conducted by Snodgrass and Vanderwart (1980), researchers were forced to rely on their own sets of pictures to design experiments, since no standardized database was available. The consequence was that the comparability of the results between studies was not optimal. To remedy this situation, Snodgrass and Vanderwart provided normative data for American English for a set of 260 black-and-white drawings. Four variables relevant for studies of perception, memory, and language were considered: name agreement (NA), image agreement (IA), conceptual familiarity (Fam), and VC.

Since the pioneering work conducted by Snodgrass and Vanderwart (1980), other picture databases have been pub- 
lished for different populations and language communities. Berman, Friedman, Hamberger, and Snodgrass (1989) collected norms for 5- to 6-year-old children, and Cycowicz, Friedman, Rothstein, and Snodgrass (1997) collected norms for 8- to 10-year-old children. Norms for pictures have been collected for adults in British English (Barry, Morrison, \& Ellis, 1997; Vitkovitch \& Tyrrell, 1995), Spanish (Sanfeliu \& Fernandez, 1996), Dutch (Martein, 1995), and more recently, French (Alario \& Ferrand, 1999), Icelandic (Pind, Jonsdottir, Tryggvadottir, \& Jonsson, 2000), and Italian (Dell' Acqua, Lotto, \& Job, 2000). As far as French is concerned, Alario and Ferrand (1999) collected norms for 400 line drawings taken from Cycowicz et al., which included the 260 line drawings from Snodgrass and Vanderwart (1980). As was pointed out by Sanfeliu and Fernandez, language-specific norms are important since, for example, the way names are used to refer to pictures has been shown to vary across languages. However, normative data for pictural stimuli are still sparse and much less numerous than those for verbal stimuli. Researchers are, therefore, often faced with the problem of finding pictorial stimuli. Indeed, researchers generally start to design an experiment by selecting verbal stimuli varying on one or several variables of interest, such as, for instance, word frequency or phoneme-to-grapheme consistency. Unfortunately, it is not often possible to find the corresponding pictures among available sets of standardized pictures. Consequently, researchers are forced to use new pictures taken from clip art libraries, children's books, or dictionaries or drawn by an artist. One problem is that norms for these pictures are generally not made available and they concern a rather limited set of pictures corresponding to the experimental stimuli. Moreover, such a situation is self-perpetuating when subsequent experiments are designed. Therefore, in an attempt to increase the pool of pictures available for experiments relying on picture stimuli, we decided to provide a new set of 299 pictures and norms for these pictures corresponding to concepts that are not already represented in the Cycowicz et al. database. Thus, our goal was to obtain a normative database for pictorial material that would be useful for future research involving French adults.

To avoid repeating already standardized pictures, $\mathrm{Cy}$ cowicz et al.'s (1997) database was chosen as the starting point for the selection of the concepts for which pictures needed to be found. Because we thought that researchers should be able to select French material from both our normative database and Alario and Ferrand's (1999) database, we had to provide pictures that adhered as closely as possible to the style used in Snodgrass and Vanderwart's (1980) pictures. The selected pictures consisted of 299 simple black-and-white line drawings (available in electronic format at http://leadserv.u-bourgogne.fr/bases/pictures). For this new set of pictures, we then collected norms for NA, IA, image variability (Ivar), Fam, VC, and AoA. To allow the simultaneous use of both databases when stimuli were selected, we also closely followed the procedures used by Alario and Ferrand (note that these authors themselves closely followed the procedures used by Snodgrass \& Vanderwart).

For each picture, norms were collected for NA, IA, Ivar, Fam, VC, and AoA. As we will set out briefly below, several studies have indicated that these variables exert a strong influence in a variety of cognitive tasks. In addition, naming latencies were collected for the entire set of pictures, and correlational/multiple-regression analyses were performed, using the above-mentioned variables as predictors.

NA refers to the degree to which participants agree on a particular name to refer to a picture. It is measured by considering the number of alternative names given to a particular picture across participants. Two measures of NA are generally computed: the percentage of participants producing the most common name and the information statistic $H$ (Shannon, 1949). According to Snodgrass and Vanderwart (1980), the $H$ value captures more information about the distribution of names across participants than does the percentage of agreement measure. NA is an important determinant of naming speed (Barry et al., 1997; Bonin, Chalard, Méot, \& Fayol, 2002; Ellis \& Morrison, 1998; Gilhooly \& Gilhooly, 1979; Lachman, 1973; Lachman, Shaffer, \& Henrikus, 1974; Paivio, Clark, Digdon, \& Bons, 1989; Snodgrass \& Yuditsky, 1996; Vitkovitch \& Tyrrell, 1995), and it has been suggested that it is the key variable responsible for the longer naming times for pictures than for words (Ferrand, 1999).

IA refers to the degree to which the mental images formed by participants in response to a picture name match the picture's appearance. Pictures that are rated high on the IA scale are responded to faster in spoken (Barry et al., 1997) and in written picture naming (Bonin et al., 2002). Ivar indicates whether the name of an object evokes few or many different images for that particular object. A strong independent effect of Ivar on spoken and written naming latencies was recently observed by Bonin et al. (2002). Studies of memory have also shown that words that are highly rated for imagery are remembered the best (Gisselbrecht, 1988; Paivio, 1969).

Fam refers to the familiarity of the depicted concept. This variable has not been found to contribute to naming times in a systematic and robust manner (Ellis \& Morrison, 1998; Jolicœur, 1985). For instance, Ellis and Morrison reported a significant effect of Fam in spoken picture naming in a multiple regression analysis, using Lorch and Myers's (1990) procedure, but not in a conventional simultaneous multiple regression. However, Hirsh and Funnell (1995) have found that patients with progressive semantic dementia achieved better naming performance for objects having a high level of Fam than for objects having low Fam (see also Feyereisen, Van der Borght, \& Seron, 1988 , on aphasic patients). Finally, Fam has been found to have a major effect on learning and memory performance (Baddeley, 1990).

$\mathrm{VC}$ corresponds to the number of lines and details in the drawing. The $\mathrm{VC}$ of the pictures has not been found to contribute robustly to naming times (Barry et al., 1997; Bonin et al., 2002; Cycowicz et al., 1997; but see Ellis \& 
Morrison, 1998). However, this variable has been found to affect ease of recognition in tachistoscopic tasks (e.g., Hartje, Hannen, \& Willmes, 1986).

A growing body of evidence also indicates that AoA is an important determinant of performance in various lexical processing tasks (Barry et al., 1997; Bonin et al., 2002; Bonin, Fayol, \& Chalard, 2001; Bonin \& Méot, 2002; Brown \& Watson, 1987; Carroll \& White, 1973a; Coltheart, Laxon, \& Keating, 1988; Gerhand \& Barry, 1998; Gilhooly \& Logie, 1981; Hodgson \& Ellis, 1998; Lachman, 1973; Lachman et al., 1974; Morrison, Chappell, \& Ellis, 1997; Morrison, Ellis, \& Quinlan, 1992; Yamazaki, Ellis, Morrison, \& Lambon Ralph, 1997). Carroll and White (1973b) have claimed that all or part of the differences in spoken picture-naming speed that are attributed to word frequency might actually be related to differences in AoA. Some researchers have therefore cast considerable doubt on whether frequency effects are genuine frequency effects or whether they should be attributed to AoA (Morrison et al., 1992). Nevertheless, other studies have reported significant effects of both word frequency and AoA (Barry et al., 1997; Lachman, 1973; Lachman et al., 1974; Morrison \& Ellis, 2000; Snodgrass \& Yuditsky, 1996). Two different objective estimates of printed word frequency (expressed as the number of occurrences per million) are provided in the database. The first corresponds to word frequency as found in Imbs (1971), and the second refers to the Frantext counts as reported in the LEXIQUE database (New, Pallier, Ferrand, \& Matos, 2001). In the following sections, we start by describing the methodology used to collect the normative and naming latency data. We then provide the normative data and discuss the relation between the six variables considered and naming latencies.

\section{METHOD}

\section{Participants}

A total of 120 psychology students participated in the rating tasks in order to fulfill a course requirement. Six groups of 20 participants each were involved in each of the six rating tasks. The participants (6 males and 114 females; mean age, 20 years; range, 17-33 years) were all native speakers of French and had normal or corrected-tonormal vision. All the separate rating tasks were performed collectively. Finally, 30 additional participants taken from the same pool ( 2 males and 28 females; mean age, 19.2 years; range, $18-23$ years) performed a standard naming task on the 299 pictures.

\section{Material}

In order to select the final set of pictures to be used in the different norming tasks, a preliminary selection phase was performed, using a pool of about 700 pictures that, for the most part, corresponded to concepts for which no pictorial representation was available in Alario and Ferrand's (1999) database.

The preliminary selection was performed by the authors. To be included in the database, a picture had to be nonambiguous and typical of the intended concept. The drawing had to include all the details necessary in order to conform to the real complexity of the object, and, as far as possible, it had to closely conform to the style of Snodgrass and Vanderwart's (1980) drawings. For each of the above criteria, the authors were given a 3-point scale. For instance, in the typicality judgment task, a value of 1 corresponded to typical, and a value of 3 to not typical, with 2 corresponding to in between.
Pictures were chosen for inclusion in the norming study if all the authors agreed on all the criteria. On the basis of these criteria, 299 pictures were chosen. Although our goal was to provide norms for concepts and their names for which no pictorial representation was available in Alario and Ferrand's (1999) database, it appeared that 9 pictures elicited the same modal names in the two databases. The overlap between the two databases is thus extremely minimal (1.29\%).

The objects belonged to 29 distinct semantic categories - that is, animal body part, artificial/imaginary creature, bird, building (or part of building), clothing (or part of clothing), container/receptacle, desk and writing material, food/fruit, house object/furniture, human body part, insect, jewel, kitchen utensil, mammals/four-footed animals, measurement instruments, media communication instrument, mollusc, musical instrument, natural element, occupation, person, plant (and part of plant), sports object, sportsman, tool, toy/game, vegetable, vehicle (and part of a vehicle), or weapon. An additional ad hoc category (i.e., mixed) was created by including objects that were difficult to categorize unambiguously. The semantic category corresponding to each picture is provided in the file, together with the norms (downloadable with the corresponding pictures at the URL mentioned above). ${ }^{1}$

\section{Procedure}

Rating tasks. The rating tasks closely followed the procedures adopted by Alario and Ferrand (1999). At the beginning of each task, the instructions both were provided in writing on a separate sheet of paper and were read aloud by the experimenter. The participants were instructed to perform each task carefully and consistently. Individual answer sheets were prepared for each rating task. For NA, IA, Fam, and VC tasks, the pictures were projected onto a large white screen by means of an overhead projector. As in Alario and Ferrand, the written modal name corresponding to the concept appeared on the individual answer sheets for the AoA and the Ivar tasks. For all the rating tasks except NA, 5-point scales were used. Great care was taken to explain to the participants that the full range of scale values had to be employed, and not only the extremes.

In the NA task, the participants had to identify each picture by using the first name (which could sometimes consist of more than one word) that came to mind, and they were asked to write it down on the answer sheet. If they could not identify the picture name, they had to indicate whether it was because they did not know the object (they had to write down DKO), they did not know the name of the object (DKN), or they were in a tip-of-the-tongue state (TOT).

In the IA task, the participants had to rate how closely each picture resembled their own mental images of the object. To this end, the experimenter spoke aloud the modal name corresponding to the picture prior to its presentation, waited for $5 \mathrm{sec}$, and then presented the picture on the screen. During the 5-sec period, the participants had to generate mental images corresponding to the name spoken aloud while closing their eyes or looking at the blank screen. Once the picture was displayed, they had to rate, on a 5-point scale, the degree of match between the picture and their generated mental images, with 1 corresponding to low agreement and 5 to high agreement.

In the Fam task, the participants were asked to evaluate the familiarity of the concept depicted by each picture according to how usual or unusual the object was in their realm of experience. Fam was defined as "the degree to which you come into contact with or think about the concept." Care was taken to explain to the participants that the rating had to be attributed to the concept itself, and not to the way it was represented. The participants answered by using a 5 -point scale, with 1 corresponding to a very unfamiliar object and 5 corresponding to a very familiar object.

In the VC task, the participants were told to evaluate the VC of each drawing, and not that of the object it represented. An answer of 5 corresponded to very complex drawing, and 1 to very simple drawing. The $\mathrm{VC}$ was defined as the number of details or the intricacy of the lines in the drawing. 


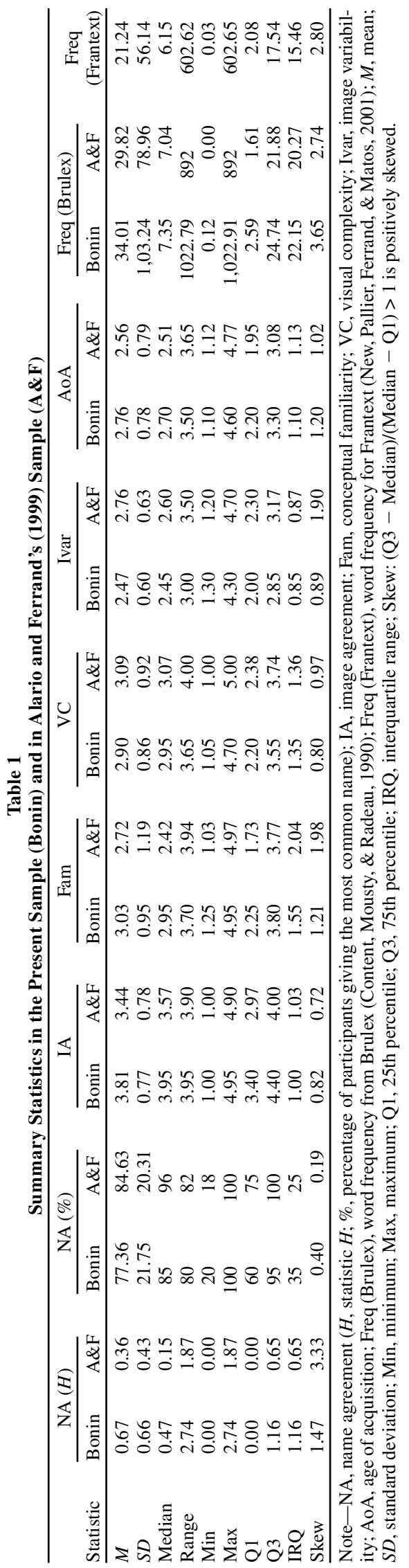

For the Ivar and AoA tasks, the ratings were performed on the basis of the written modal names. A booklet containing all the modal names was prepared. A 5-point scale was printed below each modal name. In the Ivar task, the participants had to indicate, on the 5-point scale, whether the name evoked few different mental images (rated 1) or many different mental images (rated 5). In the AoA tasks, the participants had to estimate the age at which they thought they had learned each of the names in its written or oral form. For this task, the five values of the scale corresponded to 3-year age bands with $0-3$ at one extreme and $12+$ at the other. The values were then converted to numerical values, with $1=$ learned between 0 and 3 years and $5=$ learned at age 12 or after.

Real-time naming task. The participants were tested individually in a soundproof room. They sat in front of the computer screen at a distance of about $60 \mathrm{~cm}$. Each picture was presented centered on the screen. The participants had to pronounce, as quickly as possible, the name of each picture and had to avoid making any noise before the response. They were told to tell the experimenter when they did not know the name of the picture. When they felt they knew its name but were not able to retrieve it immediately, they had to say aloud "tip of the tongue." The experimenter monitored the participants' responses and scored them for correctness. Each trial consisted of the following sequence of events. A ready signal (“*”) appeared on the screen for $500 \mathrm{msec}$ and was followed $200 \mathrm{msec}$ later by the picture. The next trial began $2,500 \mathrm{msec}$ after the participant had initiated his or her response. A short break was given to the participant after about every 45 trials. The experiment was run using PsyScope (Cohen, MacWhinney, Flatt, \& Provost, 1993) on a PowerMacintosh. The computer controlled the presentation of the pictures and recorded the latencies. An AIWA CM-T6 small tie-pin microphone connected to a button box was used to record the spoken latencies. Fifteen pictures were used as warm-ups and also to adjust microphone settings.

\section{RESULTS AND DISCUSSION}

\section{Rating Data}

The mean ratings collected for each stimulus are included in a computer file available electronically on the Internet (at the URL mentioned above). The items are listed alphabetically and are accompanied by an identifying number indicating the corresponding pictorial representation. The following information is provided for each item: (1) the most common (modal) name (together with both the intended name in parentheses, whenever it was different from the modal name, and an approximate English translation of the modal name); (2) two measures of NA corresponding to the percentage of participants giving the most common name and the $H$ statistic (as described in Snodgrass \& Vanderwart, 1980); (3) the means and the standard deviations for IA, Fam, VC, Ivar, and AoA ratings. The word frequency values (taken from Content, Mousty, \& Radeau, 1990, and New et al., 2001) of the modal name are also provided whenever available. The abbreviation NA (i.e., not available) is used to indicate that the single name (modal name) was not listed in the Content et al. corpus or in the New et al. corpus. The various nonmodal namings and their corresponding frequencies of occurrence are also provided for each of the pictorial stimuli. Of the 299 pictures, 96 yielded a single name, 84 two names, 51 three names, 38 four different names, and 17 five names. More than five names were given to 13 pictures. The numbers of naming failures (DKN, DKO, TOT) are 
Table 2

Correlations Among the Measured Variables

\begin{tabular}{lcccccccc}
\hline & \multicolumn{2}{c}{ NA } & & & & & & Freq \\
\cline { 2 - 5 } & $H$ & $\%$ & IA & Fam & VC & Ivar & AoA & (Brulex) \\
\hline$\%$ & $-.916^{*}$ & & & & & & & \\
IA & $-.244^{*}$ & $.280^{*}$ & & & & & & \\
Fam & $-.163^{*}$ & $.255^{*}$ & .102 & & & & & \\
VC & $.179^{*}$ & $-.170^{*}$ & -.086 & $-.217^{*}$ & & & & \\
Ivar & -.122 & $.205^{*}$ & $-.195^{*}$ & $.203^{*}$ & -.016 & & & \\
AoA & $.220^{*}$ & $-.321^{*}$ & .087 & $-.382^{*}$ & .142 & $-.585^{*}$ & & \\
Freq (Brulex) & $.161^{*}$ & -.111 & $-.159^{*}$ & .086 & .058 & $.395^{*}$ & $-.229^{*}$ & \\
Freq (Frantex) & $.159^{*}$ & -.101 & $-.159^{*}$ & .099 & .036 & $.418^{*}$ & $-.248^{*}$ & $.966^{*}$ \\
\hline
\end{tabular}

Note-NA, name agreement $(H$, statistic $H$; \%, percentage of participants giving the most common name); IA, image agreement; Fam, conceptual familiarity; VC, visual complexity; Ivar, image variability; AoA, age of acquisition; Freq (Brulex), word frequency from Brulex (Content, Mousty, \& Radeau, 1990); Freq (Frantext), word frequency for Frantext (New, Pallier, Ferrand, \& Matos, 2001).

*Significant at $p<.01$.

given for each item. Finally, the mean naming latency for each item is given in milliseconds.

\section{Descriptive Statistics Corresponding to the Normative Data of the Present Sample}

The following descriptive statistics for the different variables appear in Table 1: NA, IA, Fam, VC, Ivar, AoA, and the two measures of objective word frequency. ${ }^{2}$

As is shown in Table 1, two measures of NA were computed: the $H$ statistic and the percentage of participants producing the modal name (\%NA). The $H$ value equals 0 when the modal name was provided by all participants. Increasing $H$ values indicate decreasing levels of NA. As was pointed out by Snodgrass and Vanderwart (1980), the heterogeneity of the names given to a picture is better indexed by the $H$ measure than by the percentage of agreement.

When compared with Alario and Ferrand's (1999) database, Table 1 indicates that (1) the mean NA score was slightly lower and was also more heterogeneous (as a result, $H$ and $\%$ NA were less positively and negatively skewed, respectively); (2) the mean IA score was higher, whereas precisely the opposite was found for Ivar (in contrast to what was observed in Alario and Ferrand, 1999, Ivar was not positively skewed); (3) the mean VC score was very similar; (4) Fam and AoA had slightly higher mean scores; (5) as far as objective word frequency (taken from Brulex; Content et al., 1990) is concerned, the mean frequency was higher, and the frequency values were more heterogeneous and also more positively skewed than in Alario and Ferrand's sample; but (6) the reverse was true regarding Frantex frequency values (taken from New et al., 2001).

With the exception of the $H$ measures, the differences were, however, small as regards the central tendency and the heterogeneity values. The most important differences concerned the skew for $H$, Ivar, and word frequency (as given by Brulex) measures. More particularly, for $H$ and word frequency, there were more extreme positive values in the current dataset than in Alario and Ferrand's (1999) sample.

Correlational analyses. Correlational analyses were performed on the data ${ }^{3}$ (see Table 2).

As was expected, the correlation between the two NA measures $(H$ and \%NA) was high and negative $(-.917)$, and the correlation between Brulex and Frantex word frequency values was high and positive (.967).

AoA was negatively correlated with Ivar, Fam, and $\%$ NA (conversely, the correlation with the $H$ measure was significantly positive). These results indicate that early acquired words tend to evoke more different pictorial representations, have a higher NA, and refer to concepts that are more familiar than late acquired words. The correla-

Table 3

Correlations in the Present Sample (Bonin) and in Alario and Ferrand's (1999) Sample (A\& F)

\begin{tabular}{|c|c|c|c|c|c|c|c|c|c|c|c|c|c|c|}
\hline & \multicolumn{2}{|c|}{$H$} & \multicolumn{2}{|c|}{$\%$} & \multicolumn{2}{|c|}{ IA } & \multicolumn{2}{|c|}{ Fam } & \multicolumn{2}{|c|}{$\mathrm{VC}$} & \multicolumn{2}{|c|}{ Ivar } & \multicolumn{2}{|c|}{ AoA } \\
\hline & Bonin & $A \& F$ & Bonin & $A \& F$ & Bonin & $A \& F$ & Bonin & $A \& F$ & Bonin & $A \& F$ & Bonin & $A \& F$ & Bonin & $A \& F$ \\
\hline$\%$ & $-.916^{*}$ & $-.952 *$ & & & & & & & & & & & & \\
\hline IA & $-.244^{*}$ & $-.343^{*}$ & $.280 *$ & $.370 *$ & & & & & & & & & & \\
\hline Fam & $-.163 *$ & $-.183^{*}$ & $.255^{*}$ & $.215^{*}$ & .102 & -.035 & & & & & & & & \\
\hline VC & $.179 *$ & .081 & $-.170 *$ & -.081 & -.086 & -.009 & $-.217^{*}$ & $-.391 *$ & & & & & & \\
\hline Ivar & -.122 & $-.255^{*}$ & $.205^{*}$ & $.317 *$ & $-.195^{*}$ & -.097 & $.203^{*}$ & $.616^{*}$ & -.016 & $-.210^{*}$ & & & & \\
\hline AoA & $.220 *$ & $.453^{*}$ & $-.321 *$ & $-.524^{*}$ & .087 & $-.140 *$ & $-.382 *$ & $-.578 *$ & .142 & $.214^{*}$ & $-.585^{*}$ & $-.654 *$ & & \\
\hline Freq (Brulex) & $.161 *$ & $-.140^{*}$ & -.111 & $.151 *$ & $-.159 *$ & -.005 & .086 & $.360 *$ & .058 & $-.136^{*}$ & $.395^{*}$ & $.340^{*}$ & $-.229 *$ & $-.367 *$ \\
\hline
\end{tabular}

Note-NA, name agreement ( $H$, statistic $H$; \%, percentage of participants giving the most common name); IA, image agreement; Fam, conceptual familiarity; VC, visual complexity; Ivar, image variability; AoA, age of acquisition; Freq (Brulex), word frequency from Brulex (Content, Mousty, \& Radeau, 1990). *Significant at $p<.01$. 
Table 4

Factor Analysis (Varimax Rotation)

\begin{tabular}{lcccrrrr}
\hline & \multicolumn{3}{c}{ Alario and Ferrand (1999) } & & \multicolumn{3}{c}{ Present Sample } \\
\cline { 2 - 3 } \cline { 6 - 7 } Variable & \multicolumn{1}{c}{1} & 2 & 3 & & 1 & 2 & 3 \\
\hline NA $(H)$ & -.331 & .761 & -.028 & & -.158 & .715 & .220 \\
IA & -.171 & -.856 & -.033 & -.151 & -.797 & .074 \\
Fam & .720 & .011 & -.468 & .461 & -.278 & -.436 \\
VC & -.111 & .013 & .955 & .027 & .026 & .925 \\
Ivar & .844 & .022 & -.158 & .847 & .040 & .009 \\
AoA & -.799 & .333 & .139 & -.788 & .172 & .234 \\
Freq (Brulex) & .655 & .011 & .065 & .621 & .346 & .200 \\
\hline
\end{tabular}

Note-NA, name agreement ( $H$ statistic); IA, image agreement; Fam, conceptual familiarity; VC, visual complexity; Ivar, image variability; AoA, age of acquisition; Freq (Brulex), word frequency from Brulex (Content, Mousty, \& Radeau, 1990).

tion between AoA and word frequency was also negative and significant, but it was smaller in size than the previously reported correlations.

The correlation between Fam and Ivar was positive. This suggests that a larger number of different mental images are associated with familiar concepts. Fam was also positively correlated with \%NA (and negatively with the $H$ statistic), indicating that familiar concepts depicted by the pictures receive a higher name agreement score than do less familiar concepts. The negative correlation between Fam and VC reveals that familiar concepts are depicted by pictures that are visually less complex. Finally, VC was negatively correlated with the \%NA (and positively with the $H$ statistic), indicating lower name agreement for visually complex pictures. The opposite was observed as regards the correlation between IA and NA (\%NA and $H)$, which suggests that high NA scores were given to pictorial stimuli that provided good matches for the participants' mental images.

As can be seen from Table 3 , the results of the analyses were mostly similar to those obtained by Alario and Ferrand (1999), although correlation values were often weaker. This was particularly true for the correlations between Fam and Ivar, Fam and frequency, and Fam and AoA, suggesting that the familiarity of the depicted concepts was less strongly related to these variables in the present dataset than in that used by Alario and Ferrand. Other important differences between the two datasets were also found as regards the correlations between the two measures of NA with AoA, as well as for the correlation between Ivar and VC. NA seems to have been less dependent on AoA, and Ivar was less dependent on VC, in the present sample than in that used by Alario and Ferrand.

Factor analysis. In order to obtain further information on the main multivariable structures of our normative database and that used by Alario and Ferrand (1999), a principal component factor analysis and a varimax rotation was performed on each sample. ${ }^{4}$ In these analyses, only the $H$ statistic for NA was considered because (1) $H$ is a more common measure of NA and (2) using both NA measures would have given too much weight to the NA variable. In each analysis, three factors were retained: two factors because two eigenvalues were above 1 and a third factor because VC was not clearly expressed on the first two factors. ${ }^{5}$ Altogether, the three factors accounted for $65 \%$ of the variance in the present sample and $72 \%$ in Alario and Ferrand's sample.

Table 4 shows the loadings of the variables in the two analyses. In the two samples, Factor 1 loads on Ivar, AoA, and word frequency. However, whereas in Alario and Ferrand's (1999) sample, Fam was also essentially expressed on this factor, in the present sample, this variable was equally expressed on the first and the third factors. The latter aspect can be explained by the lower correlations between Fam and IA, AoA, and frequency observed in the present sample. The second factor loads essentially on NA and IA in both samples. VC complexity and, to a lesser extent, Fam contribute to the third factor.

Linear congruence coefficients (e.g., Broadbooks \& Elmore, 1987) between the factors in the two analyses (Alario and Ferrand's, 1999, and ours) were computed. ${ }^{6}$

As can be seen from Table 5, the coefficients between all pairs of factors of the same order were very close to 1 , indicating that the three dimensions are similar in the two samples. Moreover, the coefficients between differentorder factor pairs were close to 0 , indicating that a dimension in one of the samples is practically independent of another dimension in the other sample. These results show that the dimensions are essentially the same in the two analyses: (1) Ivar, AoA, word frequency; (2) NA and IA; (3) VC and, again partially, Fam. The most important discrepancy relates to Fam, which is more dependent on the first dimension in Alario and Ferrand's (1999) database than in the present one. This latter result might be due to the fact that our stimuli were slightly less familiar to the participants than was the case in Alario and Ferrand.

Table 5

Linear Congruence Coefficients

\begin{tabular}{cccc}
\hline & \multicolumn{3}{c}{ Alario and Ferrand (1999) } \\
\cline { 2 - 4 } Present Sample & Factor 1 & Factor 2 & Factor 3 \\
\hline Factor 1 & .978 & -.098 & -.322 \\
Factor 2 & -.131 & .922 & .146 \\
Factor 3 & -.248 & .157 & .945 \\
\hline
\end{tabular}


Table 6

Significant Correlations $(p<.05)$ Between Naming Times and the Other Measured Variables

\begin{tabular}{ccccccccc}
\hline & NA & IA & Ivar & VC & Fam & AoA & Freq & Phons \\
\hline NT & .309 & n.s. & -.318 & .173 & -.177 & .375 & -.309 & n.s. \\
\hline
\end{tabular}

Note-NT, naming times; NA, name agreement; IA, image agreement; Ivar, image variability; VC, visual complexity; Fam, conceptual familiarity; AoA, estimated age of acquisition taken from Alario and Ferrand (1999); Freq, Frantext word frequency (log transformed) taken from New, Pallier, Ferrand, \& Matos (2001); Phons, number of phonemes; n.s., not significant.

\section{Comparison With Other Normative Data Obtained From Other Languages: American English, Spanish, Icelandic, and Italian}

Sanfeliu and Fernandez (1996) have stressed the importance of collecting language-specific normative data. Cross-linguistic comparisons are important since they make it possible to determine whether the (correlational) structure among the variables that has been found in some languages also holds true in some others. The following analyses were thus performed to compare the relationships found in our database with other normative data obtained in other languages: American English, Spanish, Icelandic, and Italian.

American English. Apart from Ivar, for which norms were not collected, Snodgrass and Vanderwart (1980) have reported the results of correlational analyses between the same set of variables as those used in the present study (note, however, that AoA scores were available for only 89 concepts). Two points are worth noting. (1) The signs of the correlations were generally the same as those found in the present study, except for the correlation between $H$ and word frequency, which was significantly negative in their database and significantly positive in ours. However, although reliable, the latter correlation was very low in the present database (.16), and the correlation between word frequency and \%NA was not reliable, which suggests a quasi-absence of a relation between NA and word frequency in the present dataset. (2) The sizes of the correlations found by Snodgrass and Vanderwart were generally higher than those observed in our database. This holds particularly true for the correlations between Fam and VC $(-.47$ vs. -.22$)$, Fam and AoA ( -.55 vs. -.38$)$, Fam and frequency (.36 vs. .09), AoA and frequency ( -.48 vs. $-.25)$, and VC and frequency ( -.18 vs. .04). These differences could be attributable to the conceptual familiarity and the frequency of the items, which were lower in the present dataset than in Snodgrass and Vanderwart, as well as to the AoA of the items, which was higher.

Spanish. For Spanish (see Sanfeliu \& Fernandez, 1996, Table 3), the mean of the absolute differences between the available correlations in the two samples (note that in the Spanish study, only significant correlations were reported and that AoA measures were not included) was about .14, suggesting important variations between the two samples. In particular, the correlations between Fam and VC $(-.459)$, Ivar (.327), and word frequency (.214) were greater than those observed in the present sample $(-.217$,
.203 , and .086 , respectively). The correlations between Fam and NA were significant in the present sample but were not significant for the Spanish sample. The correlation between IA and Fam was significant and negative in the Spanish sample $(-.155)$, whereas it was positive but not significant in the present one (.102). Also, the two measures of NA were less highly correlated in Sanfeliu and Fernandez's sample $(-.740)$ than in the present sample (-.916). Finally, no significant correlation between word frequency and Ivar was found in Spanish, whereas the observed value was .395 in our data.

Icelandic. For Icelandic, the four significant correlations reported by Pind et al. (2000) had the same signs but showed important differences in magnitude. The correlations between Fam and AoA $(-.60)$ and between Fam and word frequency (.50) were higher than those found in the present sample ( -.382 and .086 , respectively). The same held true for the correlation between AoA and word frequency $(-.58$ and -.229 , respectively).

Italian. A comparison between the correlations found in the present study and the significant correlations reported by Dell' Acqua et al. (2000) indicates (1) the same signs for the correlations (with the exception of NA and word frequency, already discussed for American English) and (2) higher magnitudes, more particularly for AoA and Fam ( -.50 vs. -.38$)$, AoA and frequency $(-.52$ vs. $-.25)$, and Fam and Frequency (.24 vs. .10).

Taken together, the comparisons with previous published picture norms clearly show (1) that the qualitative relations between the variables, as denoted by the signs of the correlations, are essentially the same in the different languages (French, American English, Icelandic, Spanish, and Italian) and picture samples. However, (2) the magnitudes of these relations vary across studies in an important way. This last observation highlights the need to collect normative data separately for each language.

Real-time naming data. Following Snodgrass and Yuditsky's (1996) recommendation, naming latencies were trimmed on the basis of item rather than participant means, since item difficulty is such a large source of variance in naming time. Trimming was accomplished by elim-

Table 7

Values of Multiple $R$ and Beta Weights for the Independent Variables in Spoken Picture Naming

\begin{tabular}{lrlrl}
\hline Variable & $\beta$ & $S E$ & $t$ & $p$ \\
\hline NA & .243 & .065 & 3.77 & .001 \\
IA & -.219 & .066 & -3.31 & .001 \\
Ivar & -.120 & .079 & -1.52 & .13 \\
VC & .095 & .066 & 1.44 & .15 \\
Fam & -.056 & .068 & -0.83 & .41 \\
AoA & .194 & .080 & 2.42 & .02 \\
Freq & -.230 & .078 & -2.95 & .001 \\
Phons & -.003 & .075 & -0.04 & .97 \\
Multiple $R$ & & \multicolumn{2}{c}{0.556} &
\end{tabular}

Note-NA, name agreement; IA, image agreement; Ivar, image variability; VC, visual complexity; Fam, conceptual familiarity; AoA, estimated age of acquisition taken from Alario and Ferrand (1999); Freq, Frantext word frequency (log transformed) taken from New, Pallier, Ferrand, \& Matos (2001); Phons, number of phonemes. 
inating all latencies greater than two standard deviations from each item's mean.

Multiple regression analyses were performed with the inclusion of naming latencies as the dependent variable and NA, IA, Ivar, VC, Fam, AoA, and word frequency (log transformed) as the independent variables. A word length variable was also included (i.e., number of phonemes). It must be stressed that the following analyses were not intended to investigate the processes responsible for the effects. These issues have been more specifically addressed in other studies (e.g., Barry et al., 1997; Bonin et al., 2002). The purpose of the following analyses was, thus, only to assess the generality of previous findings in picture naming, using the new set of pictures.

As has generally been the case in previous multiple regression analyses in which naming latencies have been used, items having a very low NA score were not included (e.g., Barry et al., 1997; Bonin et al., 2002). More specifically, items with an NA of at least $75 \%$ (taken from the norming study) were included.

Two multiple regression analyses were performed, the first with the full set of items and the second with the exclusion of items leading to less than $50 \%$ correct responses (i.e., not corresponding to the modal name of the picture, as indicated by the normative data). The two types of analyses yielded similar results, and only the one performed with the inclusion of all items (and having an NA score of $>75 \%$ ) is reported. Because it has been claimed that a word frequency effect is more likely to emerge when recent frequency measures are used (Ellis \& Lambon Ralph, 2000), only the word frequency estimations provided in the LEXIQUE database (i.e., New et al., 2001) were included as a predictor in the analyses.

Table 6 reports the significant results of simple correlational analyses between naming times and each of the eight variables. As can be seen, naming times were positively correlated with AoA and NA $(H)$ and, to a lesser extent, with VC. Negative correlations were observed between naming times and Ivar, frequency, and to a lesser extent, Fam.

The results of the multiple regression analyses appear in Table 7. As can be seen, NA $(H)$, IA, AoA, and word frequency were found to be significant determinants of naming speed. It should be noted that a similar pattern of results was found when NA-defined as the percentage of modal NA - was substituted for the $H$ statistic.

Taken together, the results of the multiple regression analyses are consistent with those reported in the literature. In line with previous studies, we found that NA is an important determinant of naming speed (Barry et al., 1997; Bonin et al., 2002; Ellis \& Morrison, 1998; Gilhooly \& Gilhooly, 1979; Lachman, 1973; Lachman et al., 1974; Paivio et al., 1989; Snodgrass \& Yuditsky, 1996; Vitkovitch $\&$ Tyrrell, 1995). IA has not previously been included very frequently as a predictor of naming speed in picture-naming studies, but it happens to be a significant determinant of naming latencies, as has been reported by Barry et al. (1997) in spoken naming and by Bonin et al. (2002) in both spoken and written picture naming. The finding of a reliable contribution of AoA in predicting naming speed adds further evidence for the claim that lexical representations are accessed faster when words are acquired early in life than when they are acquired later. In contrast to some picture-naming studies that have failed to find reliable effects of word frequency when AoA was also taken into consideration (Barry, Hirsh, Johnston, \& Williams, 2001; Bonin et al., 2002), we found a reliable contribution of word frequency. It is worth stressing that the finding of a word frequency effect in addition to an AoA effect in picture naming in the present database cannot be attributable to the use of up-to-date word frequency measures (as was suggested by Ellis \& Lambon Ralph, 2000). In effect, in another picture-naming study that used around 200 pictures taken from Snodgrass and Vanderwart's (1980) database (Chalard, Bonin, Méot, Boyer, \& Fayol, in press), we did not find a significant contribution of word frequency in addition to a reliable AoA contribution when the recent word frequency measures (i.e., Frantext) were introduced in the multiple regression analyses. Clearly, the identification of the conditions that lead to the observation of both word frequency and AoA effects in picture naming is an issue that requires further investigation. We did not find that either Fam or VC made a significant contribution in predicting naming times. As was already stated in the introduction, these variables have not been found to contribute robustly to naming times (Barry et al., 1997; Bonin et al., 2002; Cycowicz et al., 1997; Ellis \& Morrison, 1998; Jolicœur, 1985). Finally, in contrast to a previous report of a reliable contribution of Ivar in both spoken and written picture naming (Bonin et al., 2002), we did not find a reliable contribution of this variable in the present database. The lack of an effect of this variable might be related to its lower mean and variance in the present data set.

In conclusion, the present study provides French normative data and naming times for a new set of 299 blackand-white drawings. We believe that increasing the pool of available standardized French pictorial material will be of great use to researchers involved in the study of perception, language, and memory.

\section{REFERENCES}

Alario, F.-X., \& Ferrand, L. (1999). A set of 400 pictures standardized for French: Norms for name agreement, image agreement, familiarity, visual complexity, image variability, and age of acquisition. Behavior Research Methods, Instruments, \& Computers, 31, 531-552.

BAdDeley, A. D. (1990). Human memory: Theory and practice. London: Erlbaum.

Barry, C., Hirsh, K. W., Johnston, R. A., \& Williams, C. L. (2001). Age of acquisition, word frequency, and the locus of repetition priming of picture naming. Journal of Memory \& Language, 44, 350-375.

Barry, C., Morrison, C. M., \& Ellis, A. W. (1997). Naming the Snodgrass and Vanderwart pictures: Effects of age of acquisition, frequency, and name agreement. Quarterly Journal of Experimental Psychology, 50A, 560-585.

Berman, S., Friedman, D., Hamberger, M., \& Snodgrass, J. G. (1989). Developmental picture norms: Relationships between name agreement, familiarity, and visual complexity for child and adult ratings of two sets of line drawings. Behavior Research Methods, Instruments, \& Computers, 21, 371-382.

Bonin, P., Chalard, M., Méot, A., \& Fayol, M. (2002). The determinants of spoken and written picture naming latencies. British Journal of Psychology, 93, 89-114. 
Bonin, P., Fayol, M., \& Chalard, M. (2001). Age of acquisition and word frequency in written picture naming. Quarterly Journal of Experimental Psychology, 54A, 469-489.

Bonin, P., \& MÉot, A. (2002). Spelling to dictation in real time in adults: What are the determinants of spelling latencies? Manuscript submitted for publication.

Broadbooks, W. J., \& Elmore, P. B. (1987). A Monte Carlo study of the sampling distribution of the congruence coefficient. Educational \& Psychological Measurement, 47, 1-11.

BRown, G. D. A., \& WATSON, F. L. (1987). First in, first out: Word learning age and spoken word frequency as predictors of word familiarity and word naming latency. Memory \& Cognition, 15, 208-216.

Carroll, J. B., \& White, M. N. (1973a). Age-of-acquisition norms for 220 pictureable nouns. Journal of Verbal Learning \& Verbal Behavior, 12, 563-576.

Carroll, J. B., \& White, M. N. (1973b). Word frequency and age of acquisition as determiners of picture-naming latency. Quarterly Journal of Experimental Psychology, 25, 85-95.

Chalard, M., Bonin, P., Méot, A., Boyer B., \& Fayol M. (in press). Objective age-of-acquisition (AoA) norms for a set of 230 object names in French: Relationships with other variables used in psycholinguistic experiments, the English data from Morrison et al. (1997) and naming latencies. European Journal of Cognitive Psychology.

Cohen, J., MacWhinney, B., Flatt, M., \& Provost, J. (1993). PsyScope: An interactive graphic system for designing and controlling experiments in the psychology laboratory using Macintosh computers. Behavior Research Methods, Instruments, \& Computers, 25, 257-271.

Coltheart, V., Laxon, V. J., \& Keating, C. (1988). Effects of imageability and age of acquisition on children's reading. British Journal of Psychology, 79, 1-12.

Content, A., Mousty, P., \& Radeau, M. (1990). Brulex: Une base de données informatisée pour le français écrit et parlé [Brulex: A computerized database for written and spoken French]. L'Année Psychologique, 90, 551-566.

Cycowicz, Y. M., Friedman, D., Rothstein, M., \& Snodgrass, J. G. (1997). Picture naming by young children: Norms for name agreement, familiarity, and visual complexity. Journal of Experimental Child Psychology, 65, 171-237.

Dell'Acqua, R., Lotto, L., \& Job, R. (2000). Naming times and standardized norms for the Italian PD/DPSS set of 266 pictures: Direct comparisons with American, English, French, and Spanish published databases. Behavior Research Methods, Instruments, \& Computers, 32, 588-615.

Ellis, A. W., \& Lambon Ralph, M. A. (2000). Age of acquisition effects in adult lexical processing reflect loss of plasticity in maturing systems: Insights from connectionist networks. Journal of Experimental Psychology: Learning, Memory, \& Cognition, 26, 1103-1123.

Ellis, A. W., \& Morrison, C. M. (1998). Real age-of-acquisition effects in lexical retrieval. Journal of Experimental Psychology: Learning, Memory, \& Cognition, 24, 515-523.

FERRAND, L. (1999). Why naming takes longer than reading? The special case of Arabic numbers. Acta Psychologica, 100, 253-266.

Feyereisen, P., VAn der Borght, F., \& Seron, X. (1988). The operativity effect in naming: A re-analysis. Neuropsychologia, 26, 401-415

GERHAND, S., \& BARRY, C. (1998). Word frequency effects in oral reading are not merely age-of-acquisition effects in disguise. Journal of Experimental Psychology: Learning, Memory, \& Cognition, 24, 267283

Gilhooly, K. J., \& Gilhooly, M. L. (1979). Age-of-acquisition effects in lexical and episodic memory tasks. Memory \& Cognition, 7, 214223

Gilhooly, K. J., \& Logie, R. H. (1981). Word age-of-acquisition, reading latencies and auditory recognition. Current Psychological Research, 1, 251-262.

GiSSELBRECHT, D. (1988). Concreteness encoding through a dual task procedure: Arguments in favour of an automatic process. Acta Psychologica, 67, 145-155.

Hartje, W., Hannen, P., \& Willmes, K. (1986). Effects of visual complexity in tachistoscopic recognition of Kanji and Kana symbols by German participants. Neuropsychologia, 24, 297-300.
Hirsh, K. W., \& Funnell, E. (1995). Those old, familiar things: Age of acquisition, familiarity and lexical access in progressive aphasia. Journal of Neurolinguistics, 9, 23-32.

Hodgson, C., \& Ellis, A. W. (1998). Last in, first to go: Age of acquisition and naming in the elderly. Brain \& Language, 64, 146-163.

ImBs, P. (1971). Études statistiques sur le vocabulaire français: Dictionnaire des fréquences: Vocabulaire littéraire des XIXe et XX siècle (Centre de recherche pour un Trésor de la Langue Française, CNRS, Nancy). Paris: Librairie Marcel Didier

Jolicteur, P. (1985). The time to name disoriented natural objects. Memory \& Cognition, 13, 289-303.

LACHMAN, R. (1973). Uncertainty effects on time to access the internal lexicon. Journal of Experimental Psychology, 99, 199-208.

Lachman, R., Shaffer, J. P., \& Henrikus, D. (1974). Language and cognition: Effects of stimulus codability, name-word frequency, and age of acquisition on lexical reaction time. Journal of Verbal Learning \& Verbal Behavior, 13, 613-625.

LoRCH, R. F., \& Myers, J. L. (1990). Regression analysis of repeated measures data in cognitive research. Journal of Experimental Psychology: Learning, Memory, \& Cognition, 16, 149-157.

MARTEIN, R. (1995). Norms for name and concept agreement, familiarity, visual complexity and image agreement on a set of 216 pictures. Psychologica Belgica, 35, 205-225.

Morrison, C. M., Chappell, T. D., \& Ellis, A. W. (1997). Age of acquisition norms for a large set of object names and their relation to adult estimates and other variables. Quarterly Journal of Experimental Psychology, 50A, 528-559.

Morrison, C. M., \& Ellis, A. W. (2000). Real age of acquisition effects in word naming and lexical decision. British Journal of Psychology, 91, $167-180$

Morrison, C. M., Ellis, A. W., \& Quinlan, P. T. (1992). Age of acquisition, not word frequency, affects object naming, not object recognition. Memory \& Cognition, 20, 705-714

New, B., Pallier, C., Ferrand, L., \& Matos, R. (2001). Une base de données lexicales du français contemporain sur Internet: LEXIQUE [A contemporary lexical database for French on the Internet: LEXIQUE]. L'Année Psychologique, 101, 447-462.

PAIVIO, A. (1969). Mental imagery in associative learning and memory. Psychological Review, 76, 241-263.

Paivio, A., Clark, J. M., Digdon, N., \& Bons, T. (1989). Referential processing: Reciprocity and correlates of naming and imaging. Memory \& Cognition, 17, 163-174.

Pind, J., Jonsdottir, H., Tryggvadottir, H. B., \& Jonsson, F. (2000). Icelandic norms for the Snodgrass and Vanderwart (1980) pictures: Name and image agreement, familiarity, and age of acquisition. Scandinavian Journal of Psychology, 41, 41-48.

Sanfeliu, M. C., \& Fernandez, A. (1996). A set of 254 SnodgrassVanderwart pictures standardized for Spanish: Norms for name agreement, image agreement, familiarity, and visual complexity. Behavior Research Methods, Instruments, \& Computers, 28, 537-555

Shannon, C. E. (1949). The mathematical theory of communication. In C. E. Shannon \& W. Weaver (Eds.), The mathematical theory of communication. Urbana: University of Illinois Press.

SNODGRASS, J. G., \& VANDERWART, M. (1980). A standardized set of 260 pictures: Norms for name agreement, image agreement, familiarity, and visual complexity. Journal of Experimental Psychology: Human Learning \& Memory, 6, 174-215.

SnOdgrass, J. G., \& YudiTsKY, T. (1996). Naming times for the Snodgrass and Vanderwart pictures. Behavior Research Methods, Instruments, \& Computers, 28, 516-536.

VIT Kovitch, M., \& TY RRELl, L. (1995). Sources of disagreement in object naming. Quarterly Journal of Experimental Psychology, 48A, 822-848.

Yamazaki, M., Ellis, A. W., Morrison, C. M., \& Lambon RalPh, M. A (1997). Two age of acquisition effects in the reading of Japanese Kanji. British Journal of Psychology, 88, 407-421.

\section{NOTES}

1. All the items were therefore categorized. Only the item plant seems to be dependent on a subordinate level. 
2. Note that, because of missing values, these statistics were computed using only 281 and 290 stimuli for Brulex and Frantex frequency, respectively.

3. Because of missing values, the correlations with Brulex and Frantex frequency values were computed by using 281 and 290 stimuli, respectively. The correlation between the two measures of frequency was computed on 281 stimuli.

4. These analysis were performed using only stimuli with valid values for all the variables-that is, 281 in the present dataset and 356 in Alario and Ferrand's (1999) sample.

5 . Note that the variable structures were essentially the same on the first two factors when two or three factors were used for the analyses. The only difference concerned VC, which had no clear expression on the two first factors but was well expressed on the third.
6. The linear congruence coefficient is defined by

$$
C C_{p q}=\frac{\sum_{i=1}^{n} a_{i p} b_{i q}}{\sqrt{\sum_{i=1}^{n} a_{i p}^{2} \sum_{i=1}^{n} b_{i q}^{2}}},
$$

where $n$ is the number of variables, $a_{i p}$ is the loading of the $i$ th variable on the $p$ th factor in the first sample, and $b_{i q}$ is the loading of the $i$ th variable on the $q$ th factor in the second sample. The absolute value of $C C_{p q}$ is distributed in the range of 1 (similar solutions) to 0 (total discrepancy).

(Manuscript received May 9, 2001;

revision accepted for publication June 2, 2002.) 